\title{
Expectativas de reconocimiento y estrategias de incorporación: la construcción de trayectorias degradadas en migrantes latinoamericanos residentes en la Región Metropolitana de Santiago ${ }^{1}$
}

\author{
Luis Eduardo Thayer Correa \\ Universidad de Los Lagos, Santiago, Chile. Email: luis.thayer@ulagos.cl
}

Resumen: Las expectativas de reconocimiento que tienen los migrantes puede entenderse desde la noción de falso reconocimiento, en que el sujeto se identifica con la posición desfavorable que le impone la sociedad receptora. Las expectativas de los migrantes están mediadas por la percepción de que se contrae una deuda con la sociedad en virtud de que ésta le ha entregado un espacio de oportunidades para la realización de un proyecto de vida orientado al bienestar y la seguridad. Esto redunda en una concepción del acceso a los derechos restringida y centrada en el acceso al mercado de trabajo y de bienes de consumo. En este marco, las principales estrategias de incorporación identificadas son el ocultamiento, la adaptación, la distinción al interior de los colectivos, el fortalecimiento de las redes y la priorización del trabajo.

Palabras clave: migración, reconocimiento, discriminación.

\section{Recognition expectations and incorporation strategies: the construction of degradated trayectories in latinamerican migrants residing in the Metropolitan Region of Santiago}

\begin{abstract}
Migrants recognition expectations can be understood from the notion of false recognition, in which the subject identifies with the unfavorable position imposed by the host society. The expectations of migrants are mediated by the perception that they contract a debt with the society which has given them a space of opportunities for the realization of a life project oriented to welfare and safety. This leads to a restricted conception of access to rights focused on access to labor market and consumers goods. In this context the main incorporation strategies identified are concealment, adaptation, distinction within the collectives, networks strengthening and prioritization of work.
\end{abstract}

Key words: migration, recognition, discrimination. 


\section{Expectativas de reconhecimento e estratégias de inclusão: construindo trajetórias degradadas de migrantes da América que residem na Região Metropolitana de Santiago}

Resumo: As expectativas do reconhecimento que os migrantes têm pode ser entendida a partir da noção de falso reconhecimento, em que o sujeito se identifica com a posição desfavorável imposta pela sociedade de acolhimento. As expectativas dos migrantes são mediados pela percepção de que ele incorre em uma dívida com a sociedade sob o espaço de oportunidades que esta tem dado para a realização de um projeto de vida orientado ao bem-estar e segurança. O que leva a uma concepção de acesso a direitos restrita e focada em acesso ao mercado de trabalho e bens de consumo. Neste contexto, as principais estratégias de incorporação identificadas são: a ocultação, a adaptação, a distinção dentro de grupos, o fortalimento das redes ea priorização do trabalho.

Palavras-chave: migração, reconhecimento, discriminação.

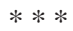

\section{Introducción}

El reconocimiento que los migrantes reciben por parte del Estado y la sociedad receptores es decisivo para definir el sentido de su trayectoria migratoria, y moldear la imagen que se construye socialmente sobre la condición de migrante. Por su parte la expectativa de reconocimiento que poseen los propios migrantes define el modo en que estos experimentan y enfrentan ese lugar y esa imagen. Asumimos con esto, como punto de partida que las trayectorias migratorias se construyen objetivamente mediante el reconocimiento institucional y social del que son objeto los inmigrantes por parte del Estado y la sociedad, y subjetivamente a partir de la forma en que estos experimentan ese reconocimiento y lo ponderan desde sus propias expectativas.

El acto migratorio es, en este sentido, una experiencia constitutiva de una nueva identidad en los sujetos que la viven (Abad, 1993), la que se define en gran medida en la relación de reconocimiento que se establece entre el migrante y el contexto (De Lucas, 2009; Cachón 2010). En nuestra investigación hemos buscado dar cuenta por un lado, del reconocimiento que de hecho reciben los inmigrantes por parte de la sociedad, y por otro, de las consecuencias prácticas esto tiene tanto para sus trayectorias como para la forma que tienen de enfrentarlas, y para la construcción de su autoimagen. La hipótesis central de nuestra investigación plantea que el reconocimiento que ofrece la sociedad receptora a los migrantes argentinos, ecuatorianos y peruanos influye en la imagen que estos construyen de sí mismos, e incide en sus estrategias de incorporación. En otra parte (Thayer, Córdova y Ávalos, 2013) hemos mostrado que el reconocimiento que ofrece la población nativa residente en la Región Metropolitana implica algunas concepciones ideológicas que condicionan la acep- 
tación. En lo que viene nos proponemos señalar los aspectos centrales que marcan la expectativa de reconocimiento de los migrantes, y las consecuencias que tiene para las estrategias que estos ponen en marcha para enfrentarlo.

La información que da cuerpo a nuestro trabajo fue generada a partir de un diseño cualitativo basado en el análisis de discurso. Para ello elaboramos dos muestras complementarias, una para la realización de entrevistas y otra para la aplicación de grupos de discusión (Ibáñez 2003). Para la primera muestra se tomaron las variables nacionalidad de origen (argentinos, ecuatorianos y peruanos) y la composición familiar (con familia dependiente en el país de procedencia, con familia dependiente en el lugar de destino, y sin familia dependiente). La justificación para considerar estas dos variables es que pensamos que marcan diferencias relevantes en cuanto a la construcción de un sentido de pertenencia a la sociedad receptora, a las estrategias de incorporación a ésta, la expectativa de reconocimiento social del que son objeto, y en cuanto al reconocimiento del que son objeto por parte de la sociedad.

En cuanto a los grupos de discusión con inmigrantes la muestra estructural también se configurará a partir de dos variables: nacionalidad de origen y tipo de autorización de residencia (definitiva u otra). Consideramos que distinguir por tipo de autorización es significativo primero porque se marca diferencias en cuanto al reconocimiento formal por parte del Estado chileno, lo que puede influir en el tipo de reconocimiento que demanden los inmigrantes. Segundo, el tipo de autorización está en directa relación con el tiempo de permanencia, lo que pensamos que influye en la intensidad que pueda tener el sentido de pertenencia a la sociedad receptora, y por tanto la definición de la expectativa de reconocimiento que tienen los inmigrantes para con ésta

En cuanto a procesamiento de la información, una vez recogida se procedió a codificarla para luego analizarla. Dada la cantidad del material empírico que recopilada fue necesario utilizar el programa Atlas.ti 5.0. El primer paso fue ordenar los discursos por los ejes temáticos generales plasmados en las pautas de entrevista y de grupos de discusión. Posteriormente se extrajeron las sub-dimensiones en cada eje, las que fueron ordenadas a partir de la asignación de códigos numéricos específicos. Una vez codificada toda la información se realizó un trabajo de reducción de los códigos, dirigida a descartar aquellos códigos que no hayan agrupado ningún fragmento de discurso o que hayan sido evaluados como poco significativos, y a fusionar los códigos con fragmentos discursivos con significado análogo y que en las pautas hayan estado pensados como dimensiones diversas. En cuanto a la aplicación tanto de entrevistas como de grupos, optamos por construir un cuestionario muy amplio y estructurado para a partir de su aplicación inicial comenzar a flexibilizarlo en función de las respuestas que se fueron generando por parte de los sujetos. El esquema general de las categorías para las pautas se sintetiza en el esquema 1. 


\section{Esquema 1: Pauta de dimensiones y categorías}

\begin{tabular}{|l|l|l|}
\hline Ámbitos del discurso & Dimensiones & Categorías transversales \\
\hline Trayectoria migratoria & - Percepción & a) Educación \\
& - Discriminación & b) Salud \\
& - Experiencia inicial, pasado & c) Estado \\
& - Experiencia actual, presente & d) Trabajo \\
& - Proyecciones y objetivos & e) Ciudad (Santiago) \\
& - Reconocimiento & f) Vivienda \\
& - Relaciones & g) Barrio \\
& - Lugar de origen & h) Autóctonos \\
& - Vinculos transnacionales & i) Paisanos \\
& & j) Otros inmigrantes \\
\hline Identidad & - Pasado & k) Redes sociales/familia \\
& - Actualidad & a) Condición de migrante \\
& - Proyecciones & b) Condición nacional \\
& & c) Condición étnica \\
& & d) Condición familiar \\
& & e) Otros grupos de referencia \\
& & f) Personal \\
\hline
\end{tabular}

\section{El reconocimiento como objeto de estudio}

Es un hecho documentado que los inmigrantes latinoamericanos residentes en Chile, con ciertos matices según condición (nacional, de clase, tiempo de residencia, etc.), se encuentran en una posición desfavorable respecto de la población nativa, en el acceso a los derechos sociales, cívicos, culturales y políticos (Martínez, 2003; 2011; Cano et. al., 2009; Stefoni, 2002; 2003; 2004; 2009; Stefoni y Fernández 2011) Siguiendo la terminología de Castles y Davidson (2000) podemos decir que los migrantes latinoamericanos en Chile viven una situación de exclusión diferencial basada en una extensión parcial de los derechos ciudadanos. Lo que conduciría a la consolidación de lo que estos autores definen como minorías étnicas (semi-excluidas, sobre-explotadas, discriminadas y culturalmente rechazadas) en lugar de llevar a la formación de comunidades étnicas (incluidas, igualitarias, respetadas y culturalmente interactivas) (op. cit.). En este marco, nos proponemos abordar el problema de las expectativas de reconocimiento de los migrantes latinoamericanos asumiendo el supuesto de su negación, al menos parcial, por parte de la sociedad.,

Nuestro problema conduce el análisis de las expectativas de reconocimiento que tienen los mirantes y las estrategias con que enfrentan el reconocimiento que ofrece la sociedad y el Estado. Dar cuenta de esta relación es de vital importancia no solo para establecer las consecuencias objetivas y subjetivas que tiene la acción de sociedad de destino para la incorporación de los migrantes, sino también para conocer las proyecciones que se pueden prever para el mediano plazo, y sugerir políticas que contribuyan a revertirla. Cuestión en el caso de Chile resulta imperiosa toda vez que se coincidimos con Stefoni (2011) en su definición de la política migratoria chilena como una política de la no política. 
En términos teóricos nuestro problema se sostiene en dos fuentes complementarias. Por una parte la teoría del reconocimiento que han desarrollado autores desde la sociología y la filosofía política (Honneth, 1997, 2006; 2009, 2010; Fraser, 2006; Taylor, 2009; Renault, 2007; Haber, 2007; De Lucas, 2007; Cachón, 2010; Fascioli, 2011; Pereira, 2011) y por otra desde los paradigmas analíticos que la sociología las migraciones ha producido a partir del estudio de los modos de incorporación de los inmigrantes a la esfera de los derechos ciudadanos (Castles y Davidson, 2000; Castles, 1995; 2003; 2006; Bauböck, 2003; 2006; Faist y Gredes, 2006; Cachón 2010; López, 2005; De Lucas, 2007; Solé 2000; Carens, 2004) y a la estructura social (Portes y Böröcz, 1989; Portes y Rumbaut 1990; Portes y DeWind, 2006).

Cabe precisar finalmente que para muchos/as investigadores/as de las migraciones internacionales, los procesos transnacionales han roto con el marco analítico del Estado nacional para el estudio de los flujos migratorios. Visto de este modo el transnacionalismo, no sería solo una opción metodológica sino un imperativo político que se levanta en oposición al nacionalismo metodológico (Beck, 2006; Canales y Montiel, 2005; Pedone, 2003; 2004) y al etnocentrismo. Si bien en nuestra investigación nos centraremos en el proceso de incorporación de los inmigrantes a la sociedad receptora, asumimos que las trayectorias y vínculos transnacionales son en la práctica complementarias y no necesariamente sustitutivas respecto de la construcción de una relación permanente con la sociedad de destino (Massey, Goldrin y Durand, 1994; Smith y Guarnizo, 1998; Smith, 1998; Vertovec, 1999; 2006; Portes, Guarnizo y Landlot, 1999; Guarnizo y Díaz, 1999; Guarnizo, Portes y Haller, 2003; Guarnizo, 2004; Levitt y Glick Schiller, 2006). En tal sentido asumimos que las expectativas de reconocimiento de los inmigrantes latinoamericanos en Santiago, pueden estar en algún sentido proyectadas a reforzar los vínculos transnacionales con los lugares de procedencia. Nuestro énfasis en los procesos de incorporación local y las influencias que ejercen sobre ellos los Estados receptores constituye un campo no solo relevante, sino también en el caso de Chile, imperioso de investigar en profundidad, y que en cualquier caso no niega la posibilidad de encontrar expectativas de reconocimiento de trayectorias transnacionales.

El análisis del reconocimiento que ofrece actualmente la sociedad a los migrantes exige distinguir los tres planos en que la bibliografía enmarca las relaciones de reconocimiento (Honneth, 1997, 2010; Honneth y Fraser, 2006; Renault, 2007; Pereira, 2011; Fascioli, 2011). Primero, el de la relaciones primarias y de los afectos básicos, segundo, el del acceso igualitario y legítimo al espacio público como sujetos políticos, y tercero, el de la valoración de la contribución que cada sujeto realiza, desde su singularidad a la reproducción material y simbólica de la sociedad. En el plano subjetivo el reconocimiento en estas tres dimensiones genera respectivamente, la auto-confianza, el auto-respeto, y la auto-valoración en la imagen que los sujetos construyen de si mismos. La negación del reconocimiento tendrá en este sentido consecuencias negativas para que los sujetos puedan realizarse en estos tres aspectos. 
En la dimensión de las relaciones primarias el desarrollo de la auto-confianza (Honneth, 1997; Renault, 2007), depende de la existencia de condiciones óptimas para la reproducción de los afectos básicos que garanticen que el sujeto pueda construir una imagen de sí mismo basada en la autoestima y la seguridad (Honneth, 1997; 2006; Fascioli, 2011). La segunda dimensión supone la aceptación de los sujetos como interlocutores válidos para la construcción del espacio público, a través de su participación en la discusión como actores legitimados para emitir juicios razonados sobre asuntos públicos (Honneth, 2010). En esta dimensión el reconocimiento se define como el acceso a la palabra y a la acción en el campo político. Para el caso de los migrantes supondría acceder al reconocimiento jurídico de derechos políticos y cívicos, y la posibilidad efectiva para ejercerlos en la sociedad de destino. El resultado del reconocimiento en esta esfera es la construcción por parte de los inmigrantes de auto-respeto. El supuesto aquí es que solo podrán respetarse los inmigrantes como sujetos públicos una vez que ellos sean reconocidos, jurídicamente y legitimados socialmente como tales, por el entorno en el que habitan.

La tercera dimensión del reconocimiento conduce a la auto-valoración y se basa en la consideración social de la contribución que cada sujeto realiza, desde su posición y condición particular a la reproducción material y simbólica de la sociedad. En este sentido el reconocimiento depende de una valoración sobre la contribución positiva a las metas consideradas valiosas por la sociedad, por lo que involucra un horizonte común de valores y objetivos (Fascioli, 2011). En el plano material la valoración se vincula a la contribución derivada del trabajo (remunerado o no) de cada cual. En el plano simbólico por su parte el reconocimiento implica una valoración de las distintas identidades grupales como parte de la cultura de la sociedad.

Sobre este último punto es necesario hacer una precisión teórica. Al tomar la concepción de reconocimiento de la formulación de Axel Honneth (1997), nos apartamos de la noción definida por Charles Taylor (2009) en su trabajo de 1990, y adoptada posteriormente por un número importante de autores (Cachón, 2010; De Lucas, 2007; Fraser, 2006). Desde esta perspectiva el problema del reconocimiento es reducido a un asunto de aceptación o no, en el espacio público de las identidades culturales diferenciadas (Fraser, 2006). La sistematización teórica de Honneth en la que inscribimos nuestro trabajo, no contradice esta idea, sin embargo circunscribe el problema de las diferencias culturales a una de las tres dimensiones del reconocimiento. Esta se configura a partir de la valoración social del aporte de los sujetos, desde su particularidad, a la reproducción material y simbólica de la sociedad. La reducción del reconocimiento al problema de la cultura es consecuencia para Honneth de un fatídico malentendido en torno a su significado (Honneth, 2010). 


\section{Las formas del reconocimiento}

La teoría asume que el reconocimiento es una relación dialógica que es constitutiva de la identidad de los agentes interactuantes (Honneth, 1997; 2006; 2010; Fraser, 2006; Taylor, 2009). Es decir, una interacción donde el individuo aprende a considerarse desde el punto de vista de los otros participantes en la interacción (Honneth, 2010). Este planteamiento recoge elementos de la filosofía política de Hegel (1999) y de la sociología pragmática de Mead (2001). Según esto la génesis del sujeto no sería monológica si no consecuencia de la relación con el otro (Taylor, 2009). El reconocimiento que el entorno dirija al sujeto es, por tanto estructurador de la imagen que este construye sobre sí mismo (Honneth, 1997). Sin embargo, la imagen que la sociedad proyecta sobre el sujeto no es completamente determinante de la que el sujeto asume como propia (op. cit.). En este punto se encuentra justamente el centro del problema que nos interesa relevar: los sujetos, en nuestro caso los migrantes, tienen la posibilidad de reaccionar al reconocimiento que ofrece el otro (Honneth; 1997; Renault, 2007). Dicho en términos de Taylor (2009) la construcción de la auto-imagen es vulnerable pero no sierva del reconocimiento. Es precisamente esta autonomía y capacidad de respuesta frente a la imagen que se construye externamente, lo que permite al sujeto reaccionar frente al reconocimiento externo, desde su propia expectativa de reconocimiento. Ello puede derivar en tres situaciones que definen la relación.

La primera se basa en una negación total o parcial del reconocimiento por parte de la sociedad y el Estado, ante la cual, el sujeto resistiría o aceptaría la relación desde el malestar. Este desajuste entre el reconocimiento recibido y el esperado supondría un agravio moral para el sujeto (Honneth, 2009) que quedaría enfrentado a una situación de menosprecio (Honneth, 1997; 2010). En este marco es que aparece el peligro de la lesión que puede sacudir la identidad de la persona en su totalidad (Honneth 1997:160). Esta negación del reconocimiento en alguna de sus tres dimensiones, al no ser aceptada por el sujeto puede motivar según Honneth, la emergencia de una lucha por el reconocimiento (op. cit.). En este sentido los migrantes vivirían su trayectoria, ya no desde una identificación con la posición subordinada en la que se insertan sino desde el malestar respecto de ella. El supuesto que asumimos en nuestra investigación es que el malestar y la frustración del inmigrante con su condición surgirá toda vez que perciba que no es reconocido del modo que él mismo aspira a serlo.

Con esta situación el Estado estaría contribuyendo a la formación de minorías étnicas (Castles y Miller, 1993) descontentas con su proceso de incorporación y excluidas al menos parcialmente del acceso igualitario a derechos. Con ello asumimos que se obstaculiza la construcción de un sentido de pertenencia de los inmigrantes a la sociedad. Si no son reconocidos como parte de esta, no podrán auto-reconocerse como parte de ella.

La segunda situación se da cuando la expectativa de reconocimiento que tiene el sujeto, coincide con el reconocimiento negado o parcial que 
ofrece la sociedad. Con ello la auto-imagen que el sujeto desarrolla quedaría inscrita en la situación de desigualdad que el Estado le impone. La identidad del sujeto se convertiría así en un instrumento de la propia dominación, o como plantea Taylor, (2009) su auto-depreciación se convierte en uno de los instrumentos más poderosos de su propia opresión (op. cit.: 54). Esta situación señalada por el autor como falso reconocimiento (op. cit.), supondría la construcción de minorías étnicas, esta vez ya no descontentas, sino resignadas y asumidas en su condición desigualitaria.

La tercera situación supone un reconocimiento auténtico (Taylor, 2009). Este implica parte del la construcción de un entorno social y normativo en el que los sujetos puedan realizarse en la dimensión de la autoconfianza, el auto-respeto y la auto-valoración. Lo que supondría eludir tanto las prácticas sociales discriminatorias y como lo que Cachón (2010) ha definido como el marco institucional discriminatorio. El reconocimiento en este sentido implica una garantía de igualdad en todos planos de la vida social como base para la incorporación de los migrantes.

La adopción de este marco teórico nos permite elaborar una estrategia analítica basada en la construcción de un modelo teórico en la lógica de los tipos ideales weberianos. Tal como lo ilustra el cuadro 1, asumimos como modelo teórico de contraste el reconocimiento auténtico que surge del cruce del reconocimiento en igualdad de condiciones, y la satisfacción de la expectativa de reconocimiento por parte de los migrantes. En la columna derecha, se encuentra el falso reconocimiento y el menosprecio. En ambos casos el Estado niega a los inmigrantes, total o parcialmente, el reconocimiento, pudiendo éstos sentir su expectativa satisfecha y no. Estas dos situaciones son las que buscamos caracterizar a partir de nuestras hipótesis.

\section{Cuadro 1: Formas de reconocimiento estatal hacia los inmigrantes}

\begin{tabular}{|c|c|c|c|c|c|}
\hline & \multicolumn{4}{|c|}{ Reconocimiento ofrecido por la sociedad y el Estado } \\
\hline & & \multicolumn{2}{|c|}{ Condiciones de igualdad y afirmación } & \multicolumn{2}{|c|}{ Condiciones de desigualdad y negación } \\
\hline \multirow[t]{2}{*}{$\begin{array}{l}\text { Expectativa } \\
\text { de los } \\
\text { sujetos }\end{array}$} & Satisfecha & $\begin{array}{l}\text { AUTÉNTICO RECONOCIMIENTO: } \\
\text { condiciones para el auto- } \\
\text { respeto, la auto-valoración y la } \\
\text { auto-confianza. } \\
\text { Comunidades étnicas } \\
\text { (reconocidas) }\end{array}$ & 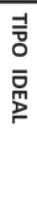 & $\begin{array}{l}\text { FALSO RECONOCIMIENTO: } \\
\text { Auto-identificación del } \\
\text { inmigrante con la posición } \\
\text { desfavorecida. } \\
\text { Minorías étnicas } \\
\text { (resignadas) }\end{array}$ & 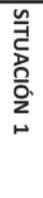 \\
\hline & Insatisfecha & \multicolumn{2}{|l|}{ (Anomalía teórica) } & $\begin{array}{l}\text { MENOSPRECIO: Lucha por el } \\
\text { reconocimiento, frustración, } \\
\text { malestar, exclusión. } \\
\text { Minorías étnicas } \\
\text { (descontentas) }\end{array}$ & 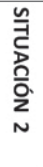 \\
\hline
\end{tabular}


En tal sentido asumimos que una situación de reconocimiento auténtico en las tres dimensiones aludidas facilita una incorporación de los inmigrantes conducente a la formación de comunidades étnicas (Castles y Miller, 1993; Castles y Davidson, 2000), mientras que el falso reconocimiento y el menosprecio serían conducentes a la formación de minorías étnicas (op. cit.). La distinción entre estas dos situaciones radicaría en la dimensión subjetiva, de las expectativas de los inmigrantes. De este modo proponemos un tipo ideal que vincula el reconocimiento a la neutralización de la relación de poder y subordinación que se da entre inmigrantes y población nativa (Renault, 2007; Haber, 2007). En las secciones que vienen se exponen los resultados centrales del análisis de discurso realizado. En primer término se presentan los significados que dan los sujetos a su condición de migrantes lo que determina el alcance que pueden tener sus expectativas de reconocimiento, luego la percepción que tienen del rechazo en sus relaciones con la población nativa y las dimensiones de la discriminación, para finalmente describir las principales estrategias de incorporación que desarrollan los migrantes latinoamericanos en la Región Metropolitana. Cierra el articulo un apartado con recomendaciones en cuanto a las políticas generadas a partir de las principales conclusiones del estudio.

\section{La condición de migrante}

La noción de migrante tiene para los tres colectivos, argentinos, ecuatorianos y peruanos un significado dual. Por una parte alude a una condición social y por otra a una condición personal. El significado que tiene la condición social de migrante es transversal a los tres colectivos y está cargada negativamente. Es un referente asociado a la precariedad de las condiciones de vida, a la discriminación social, a ocupar las posiciones más bajas en la escala ocupacional y la estructura social, y estar en una situación de exclusión social.

Frente a la condición social de migrante se pueden identificar dos posiciones divergentes influidas tanto por la distancia que los sujetos entienden que tienen con la realidad que ese referente prototípico expresa, así como por las expectativas de incorporación que tienen para el largo plazo. La medida de la identificación con la condición social de migrante es la percepción de la propia condición social. Aquellos que elaboran un relato de su experiencia migratoria basado en la precariedad, la discriminación y la subordinación tienden a aceptar la noción de migrante como un atributo que describe su identidad como sujetos sociales. Aún cuando lo enfrenten desde el malestar. Al contrario, aquellos que construyen un relato de su experiencia migratoria sobre la base del asenso social, el acceso a mejores condiciones de vida e incremento del bienestar asociado al consumo, y ausencia de experiencias discriminatorias, tienden a rechazar la noción de inmigrante como referente de su identidad social, y prefieren la de extranjero que no posee la carga negativa que imprime el entorno a la primera. 


\begin{abstract}
“Y tú te sientes parte de esta sociedad?
sí, en mucho me siento parte de aquí. Incluso cuando he tenido intercambios de palabras con gente de aquí, le digo "tú me dices que yo soy emigrante- porque ni siquiera te dicen extranjero, te dicen emigrante, que yo odio esa palabra "emigrante" porque es como tan fea la palabra “emigrante”, de que tú eres emigrante y yo soy de aquí y les digo- pero yo como porotos con riendas y tú no”. Entonces, ahí como "stop", como que se quedan pensando. "Tú eres chileno y yo no, pero yo como porotos con riendas y tú no”, y ahí los tomo, y de ahí no me dicen más nada.
\end{abstract}

\title{
¿Y por qué no te gusta la palabra “emigrante”?
}

porque se siente como tan tosca, entonces, como que un día me puse a escribir y puse "extranjero" y "emigrante” y extranjero suena como más civilizado y emigrante suena como muy tosco, como muy de abajo. Entonces, como que dije: no. No me molesta tampoco, pero sentí una diferencia, como que son muy distintas las palabras.

\section{¿Y tú crees que los chilenos cómo te ven, como extranjero o como emigrante?}

como emigrante. Entonces para mí, no sé, decir emigrante es como un curadito que anda por ahí”. (Hombre peruano)

En términos de las nacionalidades estudiadas, observamos que los peruanos aluden a la condición social de inmigrante con mayor cercanía y a la vez con mayor malestar. La perciben como más propia de su realidad social que los ecuatorianos y argentinos. Los ecuatorianos por su parte asumen esta condición social de modo heterogéneo, dándose una dualidad entre discursos de cercanía y de distancia según la experiencia pasada relatada de los sujetos. Los segmentos más acomodados y con mayor capital cultural tienden a no identificarse con la noción de migrante, al contrario de quienes se sitúan en un segmento inferior. Más homogénea y radical es la distancia que el colectivo argentino toma respecto de la condición social de migrante, a estos su significado social les resulta sumamente ajeno. La identificación con el significado de la condición social de migrante está en relación inversa con la percepción del propio bienestar. En términos comparativos podemos decir que el colectivo peruano está según su propia percepción mucho más cerca que los otros dos de la condición social de migrante.

En cuanto al significado personal de la condición de inmigrante es univoca en los tres colectivos, y está generalizada como un referente de identidad personal. La noción de migrante se asocia al desarraigo, a la capacidad de adaptación, al sacrificio, y la búsqueda de la seguridad y la estabilidad económica. El desarraigo define en términos personales la trayectoria migratoria. Aparece en el discurso como el debilitamiento de los vínculos con el lugar de procedencia y la construcción de vínculos incompletos con el de destino. La trayectoria migratoria se experimenta como un distanciamiento con el auto-reconocimiento en el lugar de origen y un reconocimiento parcial y siempre inconcluso con el de destino. En este senti- 
do la trayectoria migratoria se vive como un proceso de construcción de una trayectoria de no pertenencia. Una señal clara de esta situación la encontramos no solo el las limitaciones que perciben los migrantes para pasar a formar parte de la sociedad de destino, sino también en la distancia que sienten respecto del lugar de procedencia y la dificultad para re-construir en allí vínculos de pertenencia.

“Estar viviendo acá, a mí me pasa, me ha pasado en los últimos años de irme a mi casa y sentirme extraño en mi país, y con ganas de volver a Chile y pues claro, porque ya sabés que es tu casa; tengo mi casa, tengo mis cosas, tengo mi perro, tengo mi auto. Entonces, yo llego a la casa de MI mamá, que es allá y la voy a visitar y feliz obviamente, es mi barrio, son mis cosas, pero tengo un sentido de ya no pertenecer al lugar. Entonces, me quiero volver; quiero mi casa, quiero mi olor, quiero mis cosas. Entonces, ahí te das cuenta que en lo general, sí, te adaptaste, pero sí en realidad tomas parte, parte y posición de donde estás, que es súper importante”. (Hombre argentino)

Asociado al desarraigo los sujetos asumen que su condición de migrantes da cuenta de un rasgo personal que les permite enfrentar con éxito situaciones complejas y cambiantes. Los sujetos identifican en si mismos la capacidad para comprender nuevos códigos sociales y aprender a lidiar con ellos de manera no conflictiva. Producto de ello se definen como poseedores de una capacidad de adaptación a los cambios y a las situaciones diversas que los distingue como sujetos flexibles. Esta capacidad de adaptación, que aparece transversalmente en todos lo grupos, es interpretada como una virtud personal de los migrantes.

El sacrificio que aparece como el tercer referente personal de la condición de migrante, pasa por postergar afectos y relaciones significativos en función de acceder a una promesa de bienestar. El sacrificio migrante es entendido por los sujetos como la postergación del tiempo propio en función del desempeño en el trabajo.

\section{“Y qué cree que sea necesario que deba tener una persona para que le vaya bien acá?}

Primero, hacer la diferencia; hacer la diferencia con el resto. Si tú vienes a trabajar acá, tienes que hacer la diferencia de ser mejor trabajador, eso para cualquier extranjero, eso es un plus, porque tú te ganas la simpatía o ganas tu puesto en base al buen trabajo y eso siempre es bien valorado: la confianza, la honradez, el trabajo, la ética, la dedicación. A eso tienes que venir acá: venir con mucha energía para dedicarle a tu trabajo y demostrar que tú si vale la pena que te contraten. Eso es bueno. Tener muy buena presencia, porque acá la gente se fija en la buena presencia, entonces, la diferencia la haces tú; o sea, con tu buen trabajo, buena presencia, buena disposición, ánimo: ganarte tu espacio, y para ganarte tu espacio tienes que hacer sacrificios. Por ahí trabajar un poquito más que el otro, quedarte media hora más, no salir corriendo al tiro. Aquí a todos le 
dicen “zapatillas clavo”, son las 4 y me voy, no importa si me quedó un cliente, noo sea, entonces, cualquier argentino que venga, trate de dominar su acento, bajarse un poquito el perfil y ser un poquito más trabajador”. (Mujer argentina)

De este modo el trabajo es un referente que está fuertemente ligado a la categoría de migrante, y la que le da consistencia en relación a la experiencia del sacrificio. En este sentido la noción de migrante basada en el sacrificado realizado en la sociedad, es un referente que imprime dignidad a los sujetos a la vez que permite establecer una diferenciación respecto de los nativos. Los migrantes entienden que como trabajadores extranjeros poseen una disposición más favorable al trabajo que los chilenos, y como consecuencia de ello lo realizan de mejor manera. Este es un atributo de diferenciación basado en la condición de migrantes frente a la condición de nativos. El migrante entiende que su vida está más vinculada al trabajo que la de los nativos. Desarrollan en este sentido una ética del trabajo, tan influida por la necesidad material, como por la voluntad de lograr la autonomía futura sobre la base del sacrificio presente. La mayor pérdida personal y el mayor sacrificio que perciben que realizan los inmigrantes se da en el plano afectivo y familiar. Es una pérdida que asumen como inherente a su condición y al alejamiento de la familia que supone.

La búsqueda de la seguridad y la estabilidad económica constituye el propósito que da sentido al presente de sacrificio. Todo el sufrimiento experimentado y todo el sacrificio realizado cobran sentido y son justificados en virtud de la obtención del bienestar y la seguridad económica para el futuro. Consecuencia de ello las expectativas de reconocimiento que tienen los migrantes, está vinculada a la posibilidad de acceder a la seguridad y al bienestar.

En este sentido el acceso al consumo juega un papel más significativo que el que cumple el acceso a los derechos ciudadanos en su expectativa de reconocimiento de los migrantes. Este punto resulta central a la hora comprender la forma en que se proyectan en Chile como ciudadanos, pues tienen una concepción limitada a ciertos derechos y mediatizada por el mercado. La definición de sus derechos está fuertemente vinculada a la posibilidad de acceder en igualdad de condiciones que los chilenos a dos esferas: el mercado de bienes y el mercado laboral.

"Derechos de los extranjeros Yo creo que los extranjeros no tendrían por qué tener derechos especiales. O sea, se supone que si venís a vivir a un país, porque igual nunca escuché, por ejemplo, que si alguien se junte en una casa o en algún restaurant o algo así, a festejar su día nacional o lo que sea, que lo hayan discriminado o se lo hayan prohibido, me entendés. De hecho, ya te digo, cuando era el mundial nos juntábamos cien personas y todos con banderas y todo y no es que nadie haya venido a incendiar el local, nada más porque habían banderas argentinas o cosas así, me entendés. Entonces, esas cosas que pueden ser, la de mantener tu cultura y esas co- 
sas, hay restaurantes ecuatorianos, colombianos y todos pintados con las banderas, que podría ser igual, de una cierta forma, una falta de respeto porque si estás en Chile, por qué hay una bandera de otro país, me entendés. Creo que más que eso, no tendría por qué, me entendés, un derecho especial, porque se supone que si te vas a vivir, lamentablemente, tienes que aceptar la cultura, o las leyes que haya ahí”. (Hombre argentino)

Finalmente es necesario señalar que la percepción de los migrantes, en cuanto a que es posible acceder a estos mercados, marca sus expectativas de reconocimiento con la idea de que existe una deuda simbólica con la sociedad receptora. La apertura de un espacio de oportunidades para la realización de proyectos personales y laborales, así como la posibilidad de proyectar hacia el futuro una vida segura, motiva en los inmigrantes una disposición de agradecimiento hacia la sociedad. Esta disposición por una parte contribuye a que el migrante entienda en parte las posibilidades de acceder a las distintas dimensiones de la sociedad no tanto como un derecho adquirido sino como la concesión de una oportunidad, frente a la que se encuentra en deuda. Por otra parte como mostramos en otra parte (Thayer, Córdova, Ávalos, 2013), la población nativa entiende que los migrantes están en deuda justamente en virtud de que la sociedad les concede un espacio de oportunidades.

En tal sentido podemos concluir por una parte que hay coincidencia en cuanto a la deuda que entiende la población nativa que los migrantes tienen para con la sociedad, y la percepción de endeudamiento que los migrantes tienen respecto de ella. Al circunscribir el acceso a derechos, a esta coincidencia podemos inscribir el reconocimiento de la condición de migrante en lo que Taylor (2009) define como falso reconocimiento, esto es, la identificación de los sujetos migrantes con la posición desfavorable o no igualitaria que les impone la sociedad.

\section{Dimensiones de la discriminación y el rechazo}

Los tres colectivos perciben de manera diferencial la discriminación y consecuentemente las estrategias para enfrentarla difieren en cada grupo. Las diferencias son en general más de intensidad que de naturaleza. Vale decir son percibidas como prácticas similares que encuentran explicaciones análogas en los sujetos migrantes. Aún así el colectivo argentino es el es menos discriminación percibe, seguido por el ecuatoriano. El peruano en cambio tiende a percibir una discriminación más intensa y en más dimensiones que los otros dos.

En función de sintetizar los resultados presentaremos de manera agregada las percepciones cuidando incluir los matices diferenciadores por colectivo cuando corresponda. Los migrantes perciben que la discriminación hacia ellos se asocia a cinco condiciones: (a) la percepción de competencia en el mercado de trabajo, (b) la atribución de hábitos de convivencia 
y la intolerancia de la población nativa para acoger diferencias en este sentido, (c) la racialización por atributos físicos, (d) el ethos nacional a la enemistad, y (e) el rechazo arbitrario. En cada colectivo estos factores tienen una presencia con diferencias de énfasis.

En los contextos laborales el colectivo peruano es el que más resiente la discriminación y las manifestaciones explicitas de rechazo. En este colectivo el rechazo aparece tanto en situaciones de subordinación a empleadores o cargos superiores nativos, como en situaciones de igualdad ocupacional con nativos. Las primeras tienen sin embargo mayor presencia en el discurso y se entienden como un tipo de abuso injustificado. La discriminación de pares se interpreta al contrario en código de competencia y se justifica por una supuesta envidia en los nativos debido al mejor desempeño laboral de los peruanos. En argentinos y ecuatorianos las manifestaciones de rechazo en el trabajo tienen menor presencia en el discurso y aparecen de manera más enfática en relaciones entre pares, explicadas también por el malestar de los trabajadores nativos ante el mejor desempeño laboral de los migrantes en contextos de competencia.

\section{“¿Y por qué creen que se discrimina?}

- porque deben tener una idea diferente

- porque como en todas partes piensan que supuestamente vienen los extranjeros a quitarles el trabajo a ellos, yo he escuchado siempre, al principio era con los peruanos

- pero es que si ellos se dan cuenta, si ellos dicen que les quitan el trabajo, a ellos no les quitan el trabajo ellos hacen un trabajo que ellos no quieren hacer y lo hacen mejor

- eso, claro

- entonces ellos por decirlo así se pican y dicen eso, porque el trabajo lo hacen de mala gana, entonces viene otra persona y o hace mejor entonces ellos, se molestan en muchos tipos de trabajo, doctores, ingenieros abogados

- en la construcción se ve también, problemas o roces entre nosotros y los chilenos...porque los chilenos son buenos para dejar tirado todo, si algo no les gusta se pican y se van”. (GD ecuador)

La respuesta de los migrantes apunta a manifestar su mejor disposición para realizar el trabajo y su mejor desempeño en la realización de tareas concretas, respecto de los nativos. Esta respuesta se vincula en los colectivos ecuatoriano y peruano a la condición de migrantes. Para estos la situación migratoria los obliga tener una predisposición más favorable a aceptar las condiciones de trabajo en las que se insertan y a realizarlas con una mayor responsabilidad y exhaustividad. En el caso de los argentinos, la mejor disposición al trabajo y el mejor desempeño se asocia al nivel del educación y a un rasgo cultural propio del que carecerían, por lo general los trabajadores nativos.

El rechazo percibido por causa de los hábitos de convivencia está presente solo en el discurso de ecuatorianos y peruanos. Estos perciben que 
la población nativa los discrimina en virtud de una generalización de los hábitos y el comportamiento público de otros migrantes. Los ecuatorianos entienden que es una discriminación injustificada pues se les atribuyen a ellos hábitos propios de los inmigrantes peruanos. Para los peruanos la discriminación también resulta injustificada pues se generaliza a todos los peruanos los hábitos de unos pocos. Los hábitos a aludidos son el consumo de alcohol, los ruidos molestos y la presencia ociosa en el entorno de la vivienda o en espacios públicos específicos como parques o el centro de Santiago.

A pesar de que se genera esta transferencia a otros migrantes de las prácticas sancionadas por la población nativa, el colectivo peruano entiende que las diferencias en cuanto a las prácticas en el espacio de la vivienda y el barrio se dan en un marco de intolerancia. Los conflictos de convivencia se interpretan como consecuencia de la intolerancia de los nativos con las prácticas y hábitos de sociabilidad de los migrantes peruanos. El malestar nativo percibido por estos se atribuye también a la falta de relaciones sociales entre unos y otros. En sentido contrario ecuatorianos y argentinos perciben por una parte relaciones más intensas con los vecinos y a la vez una menor conflictividad en ese espacio.

La racialización por atributos físicos también está presente solo en ecuatorianos y peruanos, los que perciben que se los discrimina por representar la condición indígena que estaría degradada por la población nativa. Esta percepción, así como el hecho solo se haga presente en peruanos y ecuatorianos coincide con el gradiente jerárquico que va de lo blanco occidental a lo indígena americano conque la población nativa organiza su clasificación del otro latinoamericano (Thayer, Córdova y Ávalos, 2013). Las distinciones por colectivos tienen que ver esencialmente con las marcas físicas que delatan la condición de extranjero y el estatus atribuido a estos.

El rechazo por ethos nacional de enemistad aparece en el discurso del colectivo peruano y en menor medida del argentino. Está ausente en la percepción de los ecuatorianos. En los peruanos la auto-concepción como enemigos se asienta en un resentimiento anclado en la enseñanza de las relaciones históricas entre Chile y Perú. Si bien las experiencias alusivas a este tipo de rechazo no se vinculan tanto a situaciones concretas, es interpretada como uno de los factores subyacentes que limitan el desarrollo de relaciones de convivencia igualitaria. En el colectivo argentino el rechazo por enemistad nacional, aparece con menor intensidad y matizado por una concepción general de mayor aceptación de este colectivo.

Finalmente el rechazo arbitrario aparece principalmente en el colectivo peruano y en menor medida en el ecuatoriano. En el discurso de estos dos colectivos aparecen alusiones experiencias de rechazo sin motivo asociado principalmente a la primera etapa de la trayectoria migratoria. No se asocia ni a contextos ni a argumentos puntuales, sino a un malestar de la población nativa con la sola presencia del migrante. Esto lleva a que las interacciones en el espacio público se vivan por una parte como la posibi- 
lidad latente de enfrentar en cualquier momento una situación de discriminación manifiesta, y por otra como una falta de cortesía y una agresividad constantes de las interacciones.

\section{Cuadro 2: Dimensiones de la discriminación}

\begin{tabular}{|c|c|c|c|c|}
\hline Espacio & Sujeto & Rasgo del migrante & Principio & Manifestación \\
\hline La calle & Ciudadanos & Apariencia & $\begin{array}{l}\text { Racialización (impugnación } \\
\text { de diferencia inferior) }\end{array}$ & $\begin{array}{l}\text { Sospecha, desprecio } \\
\text { ofensa, agresión }\end{array}$ \\
\hline \multirow[t]{2}{*}{ Trabajo } & Compañeros & $\begin{array}{l}\text { Habilidades } \\
\text { Calificaciones }\end{array}$ & $\begin{array}{l}\text { Envidia } \\
\text { Temor a competencia }\end{array}$ & $\begin{array}{l}\text { Descalificación } \\
\text { Ridiculización }\end{array}$ \\
\hline & $\begin{array}{l}\text { Empleadores } \\
\text { Superiores }\end{array}$ & $\begin{array}{l}\text { Forma de hacer el } \\
\text { trabajo }\end{array}$ & Abuso & Maltrato, desprecio \\
\hline \multirow[t]{3}{*}{ Vivienda } & Arrendador & $\begin{array}{l}\text { Incumplimiento de pago } \\
\text { Degradación de vivienda }\end{array}$ & Prejuicio económico & \multirow[t]{2}{*}{$\begin{array}{l}\text { No arrendar } \\
\text { Arrendar más caro }\end{array}$} \\
\hline & & Malas costumbres & Intolerancia / prejuicio & \\
\hline & Vecinos & Malas costumbres & Intolerancia / prejuicio & Queja, hostilidad \\
\hline $\begin{array}{l}\text { Sistema } \\
\text { Educ. }\end{array}$ & Compañeros & $\begin{array}{l}\text { Prácticas, hábitos } \\
\text { (“cultura nacional”) } \\
\text { Trayectoria educativa }\end{array}$ & $\begin{array}{l}\text { "ethos del enemigo" } \\
\text { Prejuicio sobre formación }\end{array}$ & $\begin{array}{l}\text { Ofensa, ridiculización, } \\
\text { agresión. } \\
\text { Deslegitimación }\end{array}$ \\
\hline
\end{tabular}

En cuanto a las estrategias de enfrentamiento de la discriminación se reconocen tres dinámicas transversales a los colectivos. La primera se orienta a enfrentar las expresiones de rechazo con respuestas verbales que normalmente son contraargumentos a los argumentos discriminatorios. La segunda se dirige a ocultar las marcas que los identifican como migrantes en función de prevenir la discriminación. En los tres grupos el esconder el acento en el habla y adoptar modismos verbales locales es la táctica más común. La tercera busca generar una transferencia de la discriminación atribuyendo a otro migrante los rasgos que sirven de argumento para el rechazo. En la sección que viene presentamos una síntesis de estas estrategias de respuesta a la discriminación y otras dos estrategias que definen la trayectoria de los migrantes en la Región Metropolitana.

\section{Cinco estrategias de incorporación}

Luego del análisis del discurso de los tres colectivos, pudimos identificar cinco prácticas estratégicas que ponen en marcha los migrantes que dan cuenta de sus expectativas de reconocimiento. Cada grupo evidencia estrategias particulares y otras que son comunes a los otros dos o a alguno de ellos. El apoyo en las redes sociales, la distinción, la adaptación, el ocultamiento, y la priorización del trabajo.

Las redes sociales compuestas por connacionales son un tejido social esencial para el proceso de incorporación a la sociedad chilena. Los 
migrantes de las tres nacionalidades estudiadas las instrumentalizan de diferente modo. Un elemento en común a los tres colectivos, aunque algo menos significativo para el colectivo argentino es la existencia de un vínculo inicial con paisanos en Santiago antes de migrar. Lo cual puede vincularse a proyectos migratorios más individualizados en este colectivo, que percibe como hemos visto con una mayor valoración de su capital cultural y competencias individuales. En concordancia con esto la trayectoria de los argentinos aparece como más individualizada que la de ecuatorianos y peruanos una vez que han ingresado al país. Con este matiz, en los tres colectivos la presencia de connacionales conocidos es por lo general la puerta de ingreso a la sociedad.

Estos últimos dos grupos poseen una dependencia mayor de las redes para el acceso al mercado de trabajo, la vivienda, los códigos locales y la información administrativa y legal inicial, que los primeros. Para los argentinos las redes funcionan menos como un recurso instrumental y más como un mecanismo de reproducción de costumbres y prácticas una vez que las trayectorias se han estabilizado. Para estos la red es más un recurso de reproducción de hábitos colectivos que un recursos para el acceso material a la sociedad. La mayor valoración social de los argentinos (Thayer, Córova, Ávalos, 2013), puede explicar las estrategias individuales tengan mayor eficacia que en los otros dos colectivos en el acceso al trabajo y la vivienda. La red intragrupal como estrategia de incorporación en peruanos y ecuatorianos muestra, a su vez una debilidad de la sociedad y de Estado chileno para proveer instancias que faciliten el acceso.

La segunda estrategia de incorporación de los migrantes es la de establecer distinciones sociales, al interior de del grupo nacional en el caso de los peruanos, y respecto de los peruanos en el caso de argentinos y ecuatorianos. La distinción se utiliza como una forma de atribuir a otro los atributos identificados como más rechazados por la población nativa. La condición social del migrante precario y que constituye una carga para la sociedad, está peruanizada para los propios migrantes. En el caso de los peruanos se establece la distinción entre peruanos que constituyen un aporte a la sociedad y peruanos que no lo hacen. Estos últimos son descritos como poco predispuestos al trabajo, al ahorro y la austeridad y más propensos al desorden, el ocio y el consumo de alcohol. La imagen que se construye de este prototipo se presenta como una marca permanente. En tal sentido la podemos asociar a la noción de estigma, que Goffman (2001) define como la imposición a un sujeto de una imagen social virtual que antecede y se impone a la realidad del sujeto. La estrategia de distinción consiste, en definitiva en transferir a otro la posición inferior en función de construir y proyectar la imagen una trayectoria migratoria de contribución a la sociedad.

“-Es muy difícil porque un peruano que está bien parado aquí no va a ayudar a otro peruano.

-¿Y por qué?

-Es que acá hay mucha libertad. 


\section{-¿Cómo así?}

-Libertinaje en cierta forma, porque un peruano estando en su país es bien calladito, conservadito, pero llegando acá no. Acá viene empieza a tomar, empieza a llevar una vida que no era en su país. Por eso dicen que acá los peruanos vienen a hacer cosas que en su país no lo hacen, y eso es verdad.

\section{-¿Pero qué tipo de cosas? \\ -Empiezan a tomar, se van a fiestas, andan con una y con otra, esas son las cosas que en su país no lo hacen; y eso yo lo he visto porque tengo amigos que están acá, y en su país tenían su familia, todo era correcto. Pero acá no, acá se echaron a perder, porque acá hay mu- cha libertad. Como acá ellos vienen solos, no tienen a quien ver, tienen toda la libertad. Este es un país donde hay mucho libertinaje, cosa que a las personas que estamos acá para surgir lo vemos mal, pero nos echan al saco igual a todos. Porque yo fuera de buscar trabajo Yo tengo 28 años, y no me quieren dar trabajo porque pien- san de que porque yo soy peruana soy como dicen ustedes acá carre- tera, ando de fiesta en fiesta, y eso no es parte de mi vida”. (Mujer peruana)}

En tercer lugar, identificamos la estrategia de adaptación, que se expresa como un consenso en los tres colectivo en cuanto a la necesidad de adaptar el estilo de vida propio, al estilo de la población nativa. Existe en este sentido una sanción social hacia aquellos migrantes (de las tres nacionalidades) que mantienen las prácticas vida propias que generan desencuentros con la población nativa. Realizar esfuerzos por entender el habla local y hacerse entender por los nativos, respetar los hábitos locales en el espacio público, asumir la socialización con los chilenos como algo necesario, y respetar la normativa y el funcionamiento de las instituciones y organizaciones son las principales estrategias de adaptación.

La adaptación implica que si bien los migrantes enfrentan las diferencias de códigos con distancia, asumen que la adaptación no solo es necesaria sino también debida. En este sentido la adaptación tiene por una parte una dimensión instrumental, pues facilita el conseguir ciertos objetivos concretos, y por otra una dimensión normativa, en la que se entiende que el vivir en Santiago supone una deuda con la sociedad local a la que se responde con la cortesía de la adaptación. La apertura de un espacio de oportunidades para la realización de proyectos personales, así como la posibilidad de proyectar hacia el futuro una vida segura y estable, motiva en los inmigrantes una disposición de agradecimiento hacia la sociedad. Esta disposición contribuye a que el migrante entienda que las posibilidades de acceder a las distintas dimensiones de la sociedad no tanto como un derecho adquirido sino como la concesión de una oportunidad.

\section{"Y ¿por qué tú crees que en Chile se discriminan a los extranje-} ros que se discriminan?

Porque hay muchos. Creo que hay muchos. En mi país paso lo mis- 
mo. Llegó un momento que odiábamos a los peruanos porque se vinieron en manadas. Eran demasiadas gentes y por eso te digo que yo ahora lo veo desde otro lado. Y tenían mejores casas, tenían los mejores locales de ropa, y vos en vez de decir: pucha ay algo que quizás no estoy haciendo bien, no te gustaba esa gente. Ahora yo lo veo desde otro lado. Ahora yo soy una boliviana acá, porque yo vengo a tratar de mejorar, a tratar de estar mejor y a hacer plata, y ahora me pongo en el otro papel. Los entiendo. Me trato de adaptar y de que no nos queda otra. No siempre es bien tomado. A veces, respondido. Pero acá los invasores acá somos nosotros. ¿Sabes lo que pasa? Que pienso que uno tiene que adaptarse a donde está. No tenés por que adaptar a la gente a vos. Vos tenés que adaptarte porque vos sos el que está invadiendo. Al principio mi hija me venía con acentos de acá, y le decía no vos estás loca, la corregía, pero después no puedo hacer eso. Tu hija está viviendo acá, es natural que ella tenga acento de acá. Después fue que lo entendí y dije bueno, no puedes ser así, nos tenemos que adaptar, y así fue”. (Mujer argentina)

La cuarta estrategia de incorporación es el ocultamiento y surge como un mecanismo de prevención de la discriminación. Tiene mayor presencia en el discurso de los sujetos que no poseen marcas físicas que los tipifiquen como migrantes. En el colectivo argentino es en el que más se utiliza esta estrategia, dirigida a ocultar el acento. En estos existe una percepción de mayor similitud física respecto de la población chilena. Entre peruanos y ecuatorianos la dificultad para ocultar la condición de migrantes, plasmada más nítidamente en la fisonomía, se interpreta como una mayor exposición a la discriminación arbitraria. El ocultamiento tiene una segunda dimensión asociada a la manifestación de opiniones sobre asuntos públicos. En los tres colectivos se asume que el emitir juicios sobre asuntos públicos implica un riesgo alto de ser objeto de manifestaciones discriminatorias. Este hecho indica la falta de reconocimiento que ofrece la sociedad a los migrantes como sujetos políticos.

Finalmente, los migrantes adoptan una estrategia de priorización del trabajo como la actividad clave de su proceso de incorporación. El trabajo aparece como el eje central del discurso de los migrantes sobre si mismos. Los tres colectivos asumen que la prioridad del trabajo frente a las otras actividades de la vida cotidiana, es aquello que les permite realizar su proyecto de futuro y reproducir su trayectoria migratoria, y alcanzar la valoración de la sociedad. La dedicación al trabajo es una estrategia de afirmación de identidad migrante y de distinción respecto de la población nativa. Los migrantes por su condición de trabajadores extranjeros entienden que se ven en la obligación de trabajar más y mejor que los chilenos. Estos son percibidos como menos dependientes del trabajo y por tanto menos sometidos y menos predispuestos a realizarlo. Esta mayor dependencia del trabajo que entienden marca sus trayectorias migratorias los convierte en sujetos más vulnerables a la explotación laboral. 


\section{Conclusiones y recomendaciones}

La información recogida mediante grupos de discusión y entrevistas nos permitió identificar tres dimensiones críticas que consideramos necesarias de abordar a la hora de pensar en una estrategia de intervención pública dirigida a prevenir y reducir el rechazo de los inmigrantes latinoamericanos. En primer lugar los migrantes enfrentan una situación de vulnerabilidad al inicial sus trayectorias. Vulnerabilidad que se intensifica en los sujetos cuyos recorridos son individuales y no se inscriben en redes sociales de apoyo. La desinformación sobre los derechos reconocidos y la sujeción del permiso de residencia al contrato de trabajo con un empleador, genera que en esta etapa se experimenten situaciones de vulneración de derechos laborales. En tal sentido junto con la necesidad de apoyar la etapa inicial de las trayectorias, resulta necesario ampliar las instancias de fiscalización del trabajo en los sectores donde se concentran los migrantes e intensificar las sanciones para quienes no cumplan la normativa.

En términos normativos, pudimos identificar que el acceso al permiso de residencia definitiva entrega autonomía a los sujetos para cambiar de ocupación sin temor a perder la condición de regularidad. Facilitar el acceso a este permiso o suprimir la sujeción de la residencia al contrato de trabajo con un empleado específico es un recurso necesario, aunque no suficiente para reducir la vulnerabilidad. Si bien los migrantes que están insertos en redes sociales cuentan con un capital social que los hace menos vulnerables, en el colectivo peruano esto se ve limitado por la posición desfavorecida y por el reconocimiento precario que se encuentra a la generalidad de la red. La red migrante en este sentido da origen a un capital social limitado.

En segundo lugar, se identificó el área crítica de la vivienda. En los colectivos peruano y ecuatoriano, la precariedad habitacional se experimenta como el principal problema enfrentado una vez que las trayectorias se han estabilizado y los sujetos han accedido al permiso de residencia definitivo. Al mismo tiempo el hacinamiento y la concentración aparecen en el discurso como una causa de conflictos puntuales con vecinos nativos y como una marca que estigmatiza al conjunto de los migrantes. Se deduce de lo anterior la necesidad de facilitar el acceso a viviendas familiares, asistir con apoyo para la protección en contra de la discriminación explicita que en ocasiones excluye a los ciudadanos extranjeros como posibles arrendatarios. En la misma línea se considera necesario la implementación de programas de mediación vecinal, que resuelva y prevenga conflictos en el espacio habitacional.

En tercer lugar, se resulta necesario impulsar políticas de información y sensibilización que ponga en evidencia la realidad migratoria de la Región Metropolitana y de Chile haciendo manifiestos los aportes de la migración para la sociedad. En el discurso de los migrantes de los tres colectivos la sanción social de los nativos, aparece por lo general asociada a situaciones que se alejan de la realidad: competencia en el mercado de 
trabajo, situación generalizada de irregularidad, ausencia de espacios para los migrantes. La desinformación la falta de empatía de la población nativa con la realidad de los migrantes estimula la reproducción de prejuicios, la estigmatización y la justificación de la desigualdad de los migrantes. Una de las conclusiones presentadas a las que hemos llegado en otra parte (Thayer, Córdova y Ávalos, 2013) plantea que el contacto directo bajo ciertas condiciones contribuye a la construcción de una empatía e incluso de una identificación de la propia condición social de los nativos con la condición social de los migrantes que la comparten.

De manera que facilitar los espacios de encuentro y socialización compartida en un parco de legitimación de la presencia del migrante de la puede constituir un incentivo relevante para avanzar hacia un reconocimiento auténtico, que permita contravenir las prácticas que hoy conducen con matices a estos tres colectivos a la conformación de minorías degradadas y no de comunidades fortalecidas. 
Polis, Revista Latinoamericana, Volumen 12, $N^{\circ}$ 35, 2013

\section{Nota}

${ }^{1}$ Este artículo presenta parte de los resultados obtenidos a partir del proyecto FONDECYT Iniciación $\mathrm{N}^{\circ} 11100043$, Discriminación y lucha por el reconocimiento en el proceso de incorporación de los inmigrantes argentinos, ecuatorianos y peruanos a la Región Metropolitana. 


\section{Bibliografía}

Abad, L. V. (1993), “Nuevas formas de inmigración: un análisis de las relaciones interétnicas”, en Política y Sociedad n 12 , Madrid.

Beck, U. (2006), La sociedad del riesgo global, Siglo XXI, Madrid.

Bauböck, R. (2003), Hacia una teoría política del transnacionalismo migrante, Academia Austríaca de Ciencias.

Ídem (2006), “Migración y ciudadanía”, en Revista Zona Abierta, No 116/ 117. pp. 135-169, Madrid .

Cachón, L. (2010), La España inmigrante: marco discriminatorio, mercado de trabajo y políticas de integración, Anthropos, Barcelona.

Canales, A. y Montiel, I. (2005), ¿Un mundo sin fronteras?, Inmigración mexicana, fronteras interiores y transnacionalismo en Estados Unidos, Centro de Estudios de Población, Departamento de Estudios Regionales, Universidad de Guadalajara, Jalisco.

Carens, J. H. (2004), “La integración de los inmigrantes”, en G. Aubarell, y R. Zapata (eds.) Inmigración y procesos de cambio, Icaria, Barcelona.

Castles, S. y Miller, M. (1993), La era de la migración. Movimientos internacionales de población en el mundo moderno, INM-Camara de Diputados-Fundación Colosio, UAZ-MAP, México, D. F.

Castles, S., y Davidson, A. (2000), Citizenship and migration: globalization and the politics of belonging, Routledge, Nueva York.

Castles, S. (1995), "How Nation States respond to immigration and ethnic diversity”, en Journal of Ethnic and Migration Studies, vol 21, n³, pp. 293-308. Routledge, Londres.

Ídem (2003), “¿Adiós al multiculturalismo? Valores e identidades compartidas en las sociedades de inmigración”, en Revista de Occidente n²69, pp 22-45, Madrid.

Ídem (2006), "Factores que hacen y deshacen las políticas migratorias ”, en A. Portes y J. DeWind, Repensando las migraciones, Universidad de Zacatecas, México.

Cano, V; Soffia M. y Martínez, J (2009), Conocer para legislar y hacer política: los desafíos de Chile ante un n uevo escenario migratorio, Series Población y Desarrollo, CELADE-CEPAL, Santiago de Chile.

De Lucas, J. (2009), “Inmigración, diversidad cultural, reconocimiento 
político”, en Papers N 94, pp 53-77, Universidad Autonoma de Barcelona, Barcelona,

Faist, T. y Gredes, J. (2006), “La doble ciudadanía como un proceso dependiente de la trayectoria”, en A. Portes y J. DeWind, Repensando las migraciones, Universidad de Zacatecas, México.

Fascioli, A. (2011), "Justicia social en clave de capacidades y reconocimiento”, en ARETÉ, Revista de Filosofía, vol. XXIII, Nº1, pp 53-77, Pontificia Universidad Católica del Perú, Lima.

Fraser, N. (2006), “La justicia social en la era de la política de la identidad: redistribución, reconocimiento y participación”, en N. Fraser y A. Honnerth ¿Redistribución o reconocimiento?, Ediciones Morata, Madrid.

Guarnizo, L. E. y Díaz, L. M (1999), “Transnational migration: a view from Colombia”, en Ethnic And Racial Studies vol. 22, n 2, pp . 397-421, Routledge, Londres.

Guarnizo, L. E., Portes, A., y Haller, W. (2003), “Assimilation and transnationalism: determinants of transnational political action among contemporary migrants”, en American Journaj of Sociology, vol. 108 n $^{\circ} 6$ pp. 1211-1248, University of Chicago Press, Chicago.

Guarnizo, L. E. (2004), “Aspectos económicos del vivir transnacional”, en Escribá, A. y Rivas, N (coords.), Migración y desarrollo, CSIC, Córdoba.

Haber, S. (2007), "Recognition, justice, and social pathologies in Axel Honneths recent writings”, en Revista de Ciencia Política vol 27. N², pp 159-170, Pontificia Universidad Catolica de Chile, Santiago.

Hegel, G.F.W. (1999), La fenomenología del espíritu, FCE, México DF.

Honneth, A. (1997), La lucha por el reconocimiento. Por una gramática moral de los conflictos sociales Crítica, Grijalbo, Barcelona.

Ídem (2006), “Redistribución como reconocimiento: respuesta a Nancy Fraser”, en N. Fraser y A. Honnerth, ¿Redistribución o reconocimiento?, Ediciones Morata, Madrid.

Ídem (2009), Cítica del agravio moral. Patologías de la sociedad contemporánea, FCE, Buenos Aires.

Ídem (2010), Reconocimiento y menosprecio. Sobre la fundamentación normativa de una teoría social, Katz Editories, Buenos Aires.

Ibañez, J. (2003), Más allá de la sociología. El grupo de discusión: técnica y crítica, Siglo XXI, Madrid. 
Levitt, P. y Glick Schiller, N. (2006) "Perspectivas internacinoales sobre migración”, en A. Portes y J. DeWind, Repensando las migraciones, Universidad de Zacatecas, México.

López, A. M. (2005), Inmigrantes y Estados: la respuesta política a la cuestión migratoria, Anthropos, Barcelona.

Martínez, J. (ed.) (2011), Migración internacional en América Latina y el Caribe, CEPAL-CELADE, Santiago de Chile.

Massey, D., Goldring L. y Durand, J. (1994), “Continuities in transnational migration: an analisis of nineteen mexican communities”, en American Journal of Sociology, vol. 99, $\mathrm{n}^{\circ}$ 6, U. of Chicago Press, Chicago.

Mead, G. H. (2001), Espíritu, persona y sociedad, Paidós, Buenos Aires.

Pedone, C. (2003), Tú siempre jalas a los tuyos. Cadenas y redes migratorias de las familias ecuatorianas hacia España, Tesis inédita, U. Autónoma de Barcelona.

Ídem (2004), “Negociaciones en torno al asentamiento definitivo de las familias migrantes ecuatorianas: construcción de espacios sociales transnacionales”, en Actas de IV Congreso sobre Inmigración en España, Girona.

Pereira, G. (2011), "Eticidad y lucha por el reconocimiento: una reconstrucción de la influencia de Hegel en la democracia deliberativa”, en ARETE, Revista de Filosofía, vol. XXIII, Nº1, pp 125-158, Pontificia Universidad Católica del Perú, Lima.

Portes A. y Böröcz, J. (1989), “Contemporary immigration: theoretical perspectivas on its determinants and modes of incorporation”, en International Migration Review, vol 23, pp. 606-630.

Portes, A. y Rumbaut, R. (1990), Immigrant America: a portrait, University of California Press, Los Angeles.

Portes, A. y DeWind, J. (2006), “Un diálogo transatlántico: el progreso de la investigación y la teoría e el estudio de la migración internacional”, en A. Portes y J. DeWind, Repensando las migraciones, Universidad de Zacatecas, México.

Portes, A., Guarnizo L. E. y Landlot P. (1999), "The study of transnationalism: pitfalls and promise of an emergent research field", en Ethnic and Racial Studies, vol. 22 n $^{\circ}$ 2, pp. 217-237, Routledge, Londres.

Renault, E. (2007), "What is the use of the notion of the struggle of recognition?”, en Revista de Ciencia Política vol 27. №2, pp 195-205, Pontificia Universidad Catolica de Chile, Santiago. 
Smith, M. P. y Guarnizo L. E. (1998), “The locations of transnationalism”, en M. P. Smith, y L. E. Guarnizo (eds.), Transnationalism from below, Transaction Publishers, New Jersey.

Smith, R. (1998), “Transnational localities: community, technology and the politics of membership within the context of México and U.S. migration”, en M. P. Smith, y L. E. Guarnizo (eds.), Transnationalism from below, Transaction Publishers, New Jersey.

Solé, C. (2000), “Derechos y deberes frente al racismo y a la integración de los inmigrantes en la sociedad receptora”, en Sociedad y Utopía, ${ }^{\circ} 16$, Universidad Pontificia de Salamanca, Salamanca.

Stefoni, C. (2002), “Mujeres migrantes peruanas en Chile”, en Papeles de Población, n³3, pp, 118-145, Universidad Autónoma del Estado de México, Toluca.

Ídem (2003), Inmigración peruana en Chile. Una oportunidad de integración, Editorial Universitaria, Santiago de Chile.

Ídem (2004), “Inmigración y ciudadanía: la formación de comunidades peruanas en Santiago y la emergencia de nuevos ciudadanos”, en Rev. Política $\mathrm{n}^{\circ}$ 42, primavera, Instituto Nacional de Asuntos Públicos, U. de Chile Santiago.

Ídem (2009), “Inmigrantes en Chile. Una integración diferenciada al mercado laboral”, en VVAA Migración y políticas sociales en América Latina, SOPLA, Río de Janeiro.

Ídem (2011), “Ley y política migratoria en Chile. La ambivalencia en la comprensión del migrante”, en B. Feldman Bianco et. al. (comp.) La construcción social del sujeto migrante en América Latina. Prácticas, representaciones y categorías, CLACSO-FLACSO- U. Alberto Hurtado, Quito.

Stefoni, C. y Fernández, R. (2011), “Mujeres inmigrantes en el trabajo doméstico: entre el servilismo y los derechos”, en C. Stefoni (ed.), $\mathrm{Mu}$ jeres inmigrantes en Chile, ¿mano de obra o trabajadoras con derechos?, Ediciones Universidad Alberto Hurtado, Santiago de Chile.

Thayer, L.E, Córdova, G., Ávalos, B. (2013), “Los límites del reconocimiento; migrantes latinoamericanos en la Región Metropolitana de Santiago de Chile”, Revista Perfiles Latinoamericanos N42 pp. 163-191, FLACSO, México D.F.

Taylor, Ch. (2009), El multiculturalismo y la política del reconocimiento, F.C.E., México D. F.

Vertovec, S. (1999), “Conceiving and researching transtationalism”, en 
Ethnic and Racial Studies, vol 22, N², pp. 447-462, Oxford University Press, Oxford.

Ídem (2006), “Transnacionalismo migrante y modos de transformación”, en A. Portes y J. DeWind, Repensando las migraciones, Universidad de Zacatecas, México.

Recibido: 15.07.2013

Aceptado: 27.07.2013 